\title{
Lattice strain during compressive loading of AlCrFeNiTi multi-principal element alloys
}

Received: 1 July 2020 / Accepted: 11 February 2021 / Published online: 12 March 2021

(C) The Author(s) 2021

\begin{abstract}
In this work, multi-principal element alloys (MPEAs) with the five base elements $\mathrm{Al}, \mathrm{Cr}, \mathrm{Fe}, \mathrm{Ni}$ and Ti plus elements in minor amounts were produced by powder metallurgy and their microstructure and elastic behavior were analyzed via light and scanning electron microscopy, electron backscatter diffraction (EBSD) and synchrotron X-ray diffraction. The two studied compositions are an MPEA with Al, $\mathrm{Cr}, \mathrm{Fe}, \mathrm{Ni}$ and $\mathrm{Ti}$ in equimolar ratio as well as a similar composition with a concentration of Ti reduced to $10 \mathrm{~mol} \%$. The goal is to analyze the microstructural behavior of these compositions during macroscopic loading in dependence of chemical composition and phases present. Analysis via synchrotron X-ray diffraction predicts the presence of body-centered cubic phases, Full Heusler-phases and C14_Laves-phases in both compositions, MPEA5 and MPEA_Ti10. Synchrotron X-ray diffraction offers the possibility to monitor the deformation of these phases during macroscopic loading of specimens. Thermodynamic calculations of stable phases predicted a microstructure of MPEA5 consisting of body-centered cubic and Full Heusler-phases at room temperature. Further calculation and X-ray diffraction experiments showed the stabilization of minor amounts of C14_Lavesphase $\left(\mathrm{Fe}_{2} \mathrm{Ti}\right)$ at room temperature with a decreasing amount of Ti. MPEA5 showed the development of long and un-branched cracks during compressive testing, which resulted in a remarkable decrease in lattice-dependent elastic moduli. MPEA_Ti10 exhibited branched cracks during compression tests. Also, the lattice-dependent elastic moduli of MPEA_Ti10 did not change notably during the compression tests. In both compositions, the Full Heusler-phase showed the lowest lattice-dependent elastic moduli, hence taking the largest share of the overall deformation among all phases present in the materials under macroscopic loading.
\end{abstract}

Keywords Synchrotron X-ray diffraction - MPEA - Laves phase $\cdot$ Powder metallurgy $\cdot$ Elastic moduli

\section{Introduction}

High-entropy alloys (HEAs) and related concepts have proved their potential to provide exceptional material properties in countless research projects within the last decade [1-3]. Usually, HEAs have an equimolar ratio of

Communicated by Marcus Aßmus, Victor A. Eremeyev and Andreas Öchsner.

M. Reiberg $(\varangle) \cdot$ E. Werner

Institute of Materials Science and Mechanics of Materials, Technical University of Munich, Boltzmannstr. 15, 85748 Garching, Germany

E-mail: reiberg@wkm.mw.tum.de

X. Li

German Engineering Materials Science Centre (GEMS) at Heinz Maier-Leibnitz Zentrum (MLZ),

Helmholtz-Zentrum Geesthacht, Lichtenbergstr. 1, 85748 Garching, Germany

E. Maawad

Institute of Materials Research, Helmholtz-Zentrum Geesthacht, Max-Planck-Str. 1, 21502 Geesthacht, Germany 
at least five base elements, which results in a single-phase microstructure that is stabilized by large values of the configurational entropy. Material properties like yield strength and corrosion resistance are affected positively by a characteristically high lattice distortion in HEAs [3]. HEA-related materials are complex concentrated alloys (CCAs) and multi-principal element alloys (MPEAs).

In this work, MPEAs are produced by combining high alloyed powder materials with elemental metallic powders. The Ni-based superalloy Inconel ${ }^{8} 718$ (L718) has a high tensile strength and good creep resistance at elevated temperatures, making it an important material for components of gas turbines or liquid-fueled rockets [4-7]. The ultra-high-strength maraging steel W722 impresses with its high toughness and high tensile strength. W722 is used for shear plates and molds for the polymer industry $[8,9]$.

As has been described in a previous work, the powder materials L718 and W722 are combined with further elemental powders to form an alloy with an equimolar concentration of the elements $\mathrm{Al}, \mathrm{Cr}, \mathrm{Fe}, \mathrm{Ni}$ and $\mathrm{Ti}$ [10]. Since the compositions were partly made of L718 and W722 powder, further elements were brought into the resulting alloy system. The formation of a multi-phase microstructure is observed in the produced alloys, which leads to compositions that are designated as MPEAs instead of HEAs.

In this research, MPEAs with different chemical compositions were produced via mechanical alloying of powders and sintering via hot isostatic pressing (HIP). Two different MPEAs were produced, i.e., an alloy with equimolar concentrations of the elements $\mathrm{Al}, \mathrm{Cr}, \mathrm{Fe}, \mathrm{Ni}$ and $\mathrm{Ti}$ (MPEA5) and a composition with a reduced amount of titanium (MPEA_Ti10). Here, MPEA_Ti10 represented a first step of changing the chemical composition of MPEA5 toward the composition of L718. By stepwise approaching the chemical composition of L718, it was intended to study if positive material properties of MPEA5 and L718 can be successfully combined. Due to their very attractive material properties, the alloys are potentially suitable for load-bearing applications in the high-temperature regime fulfilling further requirements regarding wear and oxidation resistance [11-13]. To understand the macroscopic mechanical properties of MPEA5 and MPEA_Ti10, the reaction to mechanical loading of individual phases was analyzed for different reflecting planes. For this matter, cylindrical samples of the materials were tested and changes in their microstructure and phase-specific mechanical response during compression tests were analyzed via synchrotron X-ray diffraction."

\section{Experimental procedures}

\subsection{Base materials}

Through an appropriate combination of 5 different input metal powders, an MPEA with an equimolar ratio of the base elements $\mathrm{Al}, \mathrm{Cr}, \mathrm{Fe}, \mathrm{Ni}$ and $\mathrm{Ti}$ (MPEA5) as well as a composition with a reduced amount of $\mathrm{Ti}$ to 10 mol\% (MPEA_Ti10) were generated. The chemical composition of the input metal powders as well as of the materials MPEA5 and MPEA_Ti10 is listed in Table 1. The pre-alloyed powders L718 and W722 contain minor amounts of additional elements, e.g., $\mathrm{Co}, \mathrm{Mo}$ and $\mathrm{Nb}$, which are introduced into the mixture alongside the main elements $\mathrm{Al}, \mathrm{Cr}, \mathrm{Fe}, \mathrm{Ni}$ and Ti. The chemical composition of our MPEA5 is altered by changing the mixing ratio of the powders. A detailed description of the production of the samples of this study is presented in [10], and is briefly summarized in the following subsection.

\subsection{MPEA production}

A total amount of $39 \mathrm{~g}$ of powder per batch was placed into a $250 \mathrm{ml}$ tungsten carbide milling chamber combined with tungsten carbide milling balls in a ball-to-powder mass ratio of 10:1. The milling chamber was sealed under argon atmosphere in a glove box and was then placed into a planetary ball mill of the type Pulverisette P6 (Fritsch GmbH, Idar-Oberstein, Germany). The milling speed was $400 \mathrm{rpm}$ and the milling time was $2 \mathrm{~h}$. For the sake of cooling the milling chamber, five minutes of milling were alternated with 20 minutes of resting. After the milling process, only the loose powder in the milling chamber was used for further processing.

Encapsulation of the mechanically alloyed powder is required for powder densification employing hot isostatic pressing. The powder $(6 \mathrm{~g})$ with the chemical composition MPEA5 or MPEA_Ti10 is filled and sealed in Ti tubes (grade 2, $10 \mathrm{~mm}$ outer diameter, $1 \mathrm{~mm}$ wall thickness and $100 \mathrm{~mm}$ length) under argon atmosphere. A pressure of $75 \mathrm{MPa}$ and a temperature of $950^{\circ} \mathrm{C}$ were applied to the capsules by using a hot isostatic press (EPSI, Belgium) with high-purity argon gas as pressure medium. After maintaining this environment for 4 hours, the temperature is reduced moderately to room temperature with a maximum cooling rate of $12 \mathrm{~K} / \mathrm{min}$. For each composition, a sample for analysis by in situ synchrotron X-ray diffraction and 
Table 1 Chemical composition of the input material powders and the mechanically alloyed MPEA5 and MPEA_Ti10 materials

\begin{tabular}{lllllllll}
\hline Sample & $\mathrm{Al}$ & $\mathrm{Cr}$ & $\mathrm{Fe}$ & $\mathrm{Ni}$ & $\mathrm{Ti}$ & $\mathrm{Co}$ & $\mathrm{Mo}$ & $\mathrm{Nb}$ \\
\hline Al [ma.-\%] & 99.70 & 0.00 & 0.00 & 0.00 & 0.00 & 0.00 & 0.00 & 0.00 \\
Cr [ma.-\%] & 0.38 & 99.24 & 0.14 & 0.00 & 0.00 & 0.00 & 0.00 & 0.00 \\
Ti [ma.-\%] & 0.00 & 0.00 & 0.20 & 0.00 & 99.50 & 0.00 & 0.00 & 0.00 \\
W722 [ma.-\%] & 0.00 & 0.00 & 66.90 & 18.00 & 1.00 & 9.25 & 4.85 & 0.00 \\
L718 [ma.-\%] & 0.50 & 18.00 & 18.50 & 53.68 & 0.95 & 0.00 & 3.00 & 5.30 \\
MPEA5 [mol\%] & 19.21 & 19.21 & 19.21 & 19.21 & 19.21 & 1.77 & 1.11 & 0.98 \\
MPEA_Ti10 [mol\%] & 21.41 & 21.41 & 21.41 & 21.41 & 10.00 & 1.97 & 1.24 & 1.09 \\
\hline
\end{tabular}

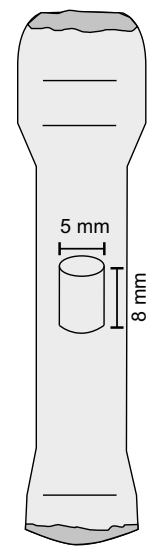

(a)

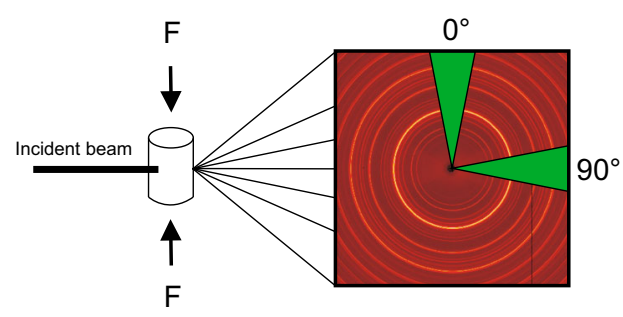

(b)

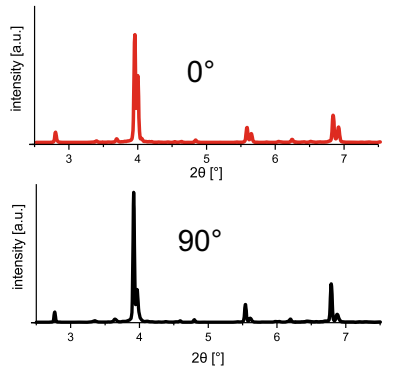

(c)

Fig. 1 Sample preparation and in situ measurements via synchrotron X-ray diffraction. a Dimensions of a cylindrical sample extracted from the Ti-capsule via WEDM. b The sample is compressed and its microstructure is analyzed via synchrotron X-ray diffraction. The detector generates diffraction images consisting of Debye-Scherrer rings. The integration of $10^{\circ}$ pie segments in $0^{\circ}$ - and $90^{\circ}$-directions for the analysis of lattice deformation in loading direction and perpendicular to it. c Output of integrated $10^{\circ}$ pie segments are 2-dimensional diffractograms which are used for further analysis

a sample for dilatometry was prepared. The amount of mechanically alloyed powder was low, resulting in a limitation of the number of specimens that could be produced.

\subsection{Dilatometry}

Compression tests were performed by using a deformation-dilatometer of type DIL 805A/D/T (TA Instruments, New Castle, USA). One test was performed for each composition. Cylindrical samples with a diameter of $4 \mathrm{~mm}$ and a length of $8 \mathrm{~mm}$ were used in these tests (Fig. 1a). The strain-controlled room temperature tests were conducted with a fixed traverse speed of $10 \mu \mathrm{m} / \mathrm{s}$.

\subsection{In situ synchrotron X-ray diffraction}

Synchrotron X-ray diffraction of the samples MPEA5 and MPEA_Ti10 combined with a compression test was performed at High Energy Materials Science (HEMS) beamline run by the Helmholtz-Zentrum Geesthacht at PETRA III at DESY in Hamburg [14]. One test was performed for each composition. Cylindrical samples with a diameter of $4 \mathrm{~mm}$ and a length of $8 \mathrm{~mm}$ were fabricated via wire electrical discharge machining (WEDM) (Fig. 1a). The samples were compressed with a constant traverse speed of $7.00 \times 10^{-4} \mathrm{~mm} \mathrm{~s}^{-1}$. The beam possesses a mean photon energy of $87.1 \mathrm{keV}$ and a wavelength of $0.14235 \AA$. A PerkinElmer XRD 1622 flat panel detector was used to analyze the diffracted beam (Fig. 1b). The detector had a pixel matrix of $2048 \times 2048$ and a pixel size of $200 \times 200 \mu \mathrm{m}^{2}$. The distance between sample and detector was set to approximately $1490 \mathrm{~mm}$ which was calibrated with an $\mathrm{LaB}_{6}$ powder standard. 


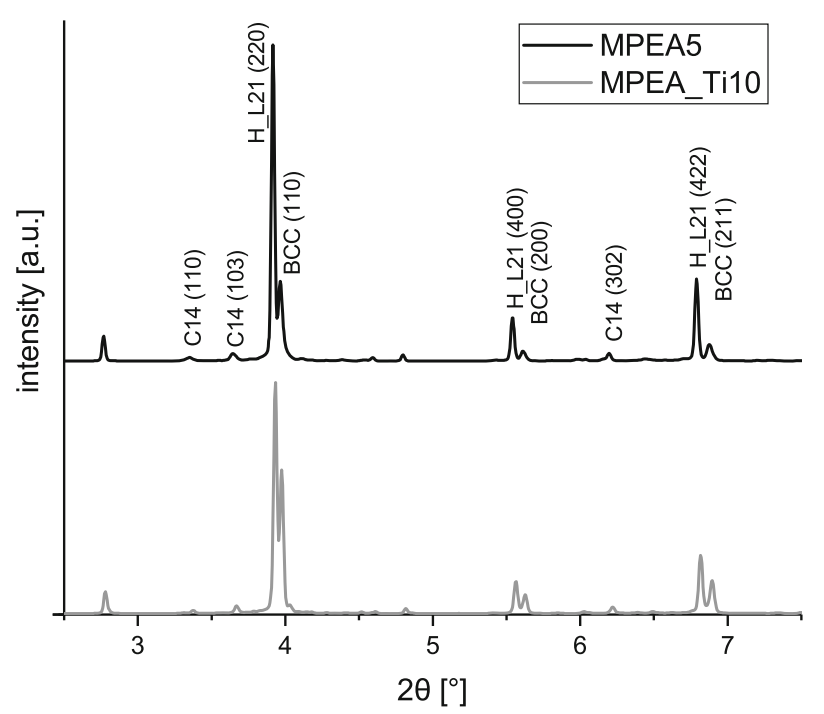

Fig. 2 Diffractograms of the compositions MPEA5 and MPEA_Ti10 obtained from samples prior to mechanical loading. Selected peaks are tagged with corresponding phases together with the reflecting lattice plane

\subsection{Strain analysis}

The radial displacement of the respective diffraction peak center with consideration of an unstrained reference peak position was determined to define the lattice parameter and the lattice strain during compression of the samples [15]. For this purpose, $10^{\circ}$ pie segments in loading direction $\left(0^{\circ}\right)$ and perpendicular to it $\left(90^{\circ}\right)$ of the two-dimensional diffraction patterns were integrated azimuthally using the software STeCa2-2.0.5RC4 (Fig. 1b, c). Three reflections per phase, which are labeled in Fig. 2, were fitted using Gaussian functions. From the position of the $i$-th peak, $\Theta_{\mathrm{hkl}, i}$, the corresponding lattice plane spacing $d_{\mathrm{hkl}, i}$ is calculated using Bragg's law: $n \lambda=2 d_{\mathrm{hkl}, i} \sin \left(\Theta_{\mathrm{hkl}, i}\right) . \lambda$ is the wavelength of the X-ray beam $(0.14235 \AA)$ and $n$ represents the order of the reflection. The elastic strains were calculated via $\varepsilon_{\mathrm{hkl}, i}=\left(d_{\mathrm{hkl}, i}-d_{\mathrm{hkl}, 0}\right) / d_{\mathrm{hkl}, 0}$. Here, $d_{\mathrm{hkl}, 0}$ represents the respective lattice plane spacing in unloaded condition. The $\sigma-\varepsilon_{\mathrm{hkl}}$ curves (see Figs. 3 and 4 ) were linearly fitted to determine the lattice-dependent elastic moduli $E_{\mathrm{hkl}}$. Thereby, each stage (I, II, III) was linearly fitted separately and the lattice-dependent elastic moduli were calculated from $E_{\mathrm{hkl}}=\left|\Delta \sigma / \Delta \varepsilon_{\mathrm{hkl}}\right|$. Further, the lattice-dependent Poisson's ratios were determined from the lattice-dependent elastic moduli in the loading direction $\left(0^{\circ}\right)$ and perpendicular to it $\left(90^{\circ}\right)$ as $\nu_{\mathrm{hkl}}=E_{\mathrm{hkl}, 0^{\circ}} / E_{\mathrm{hkl}, 90^{\circ}}$. Detailed analysis of single peaks was performed by using the software FullProf Suite vers. 07.2017 with the included tool WinPLOTR vers. 09.2018.

\subsection{Microstructure and properties analyses}

The calculation-of-phase-diagrams-method (CALPHAD) as part of the software Thermocalc along with the database TCHEA1 was used for the calculation of stable phases occurring in the alloys.

Samples for the analysis via light microscopy and electron backscatter diffraction (EBSD) were embedded in resin, ground and polished, after they have been tested via in situ synchrotron X-ray diffraction. EBSD measurements were undertaken with a Jeol JSM-7600F field emission scanning electron microscope equipped with an Oxford SYMMETRY system and AZtech software. The results were analyzed with the software Channel5 and a NIST structural database.

\section{Results and discussion}

\subsection{Macroscopic stress-strain development of MPEA5 and MPEA_Ti10}

The macroscopic stress-strain development during the in situ synchrotron compression test of composition MPEA5 is presented in Fig. 5. In the loading range between 0 and $200 \mathrm{MPa}$ different effects, e.g., settlement 


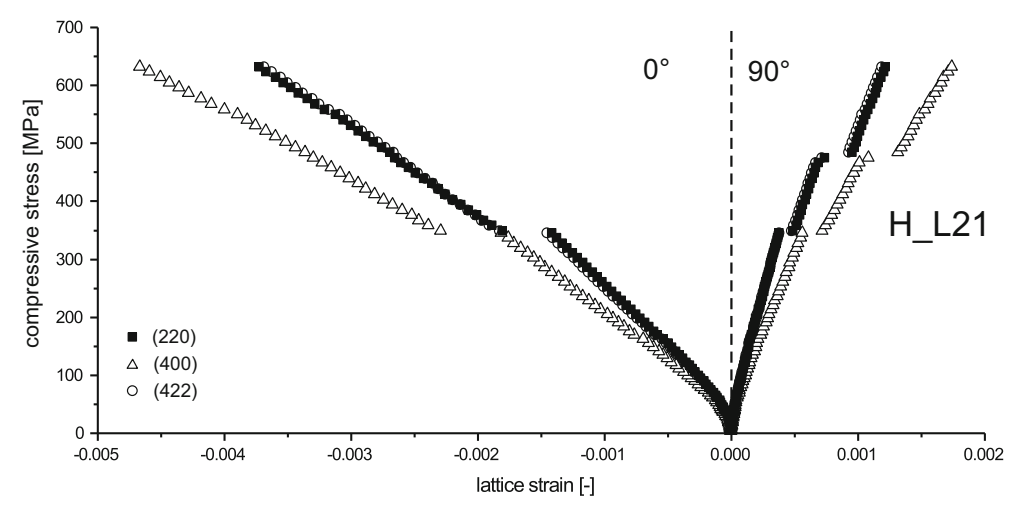

(a)

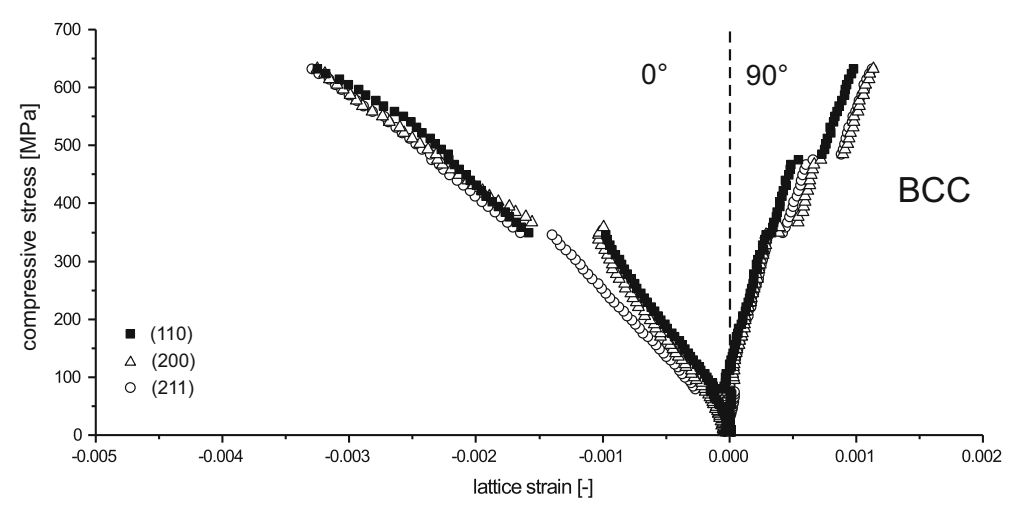

(b)

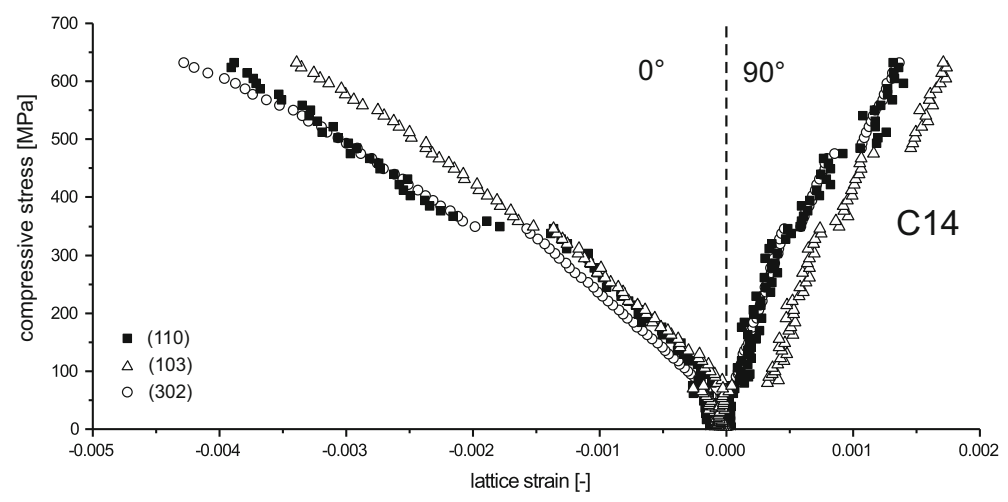

(c)

Fig. 3 Macroscopic stress plotted over lattice strain of composition MPEA5. Lattice strain is separated in parallel to loading direction $\left(0^{\circ}\right)$ and perpendicular to it $\left(90^{\circ}\right)$. The diagrams show results for the H_L21-phase (a), BCC-phase (b) and C14_Lavesphase $(\mathbf{c})$

of the sample on a microscopic scale, results in a nonlinear curve progression. In the compressive stress range from 200 to $636 \mathrm{MPa}$, the stress increase is linearly correlated to the strain increase. There are two stress levels at which the stress-strain curve exhibits a kink. The enlarged areas in Fig. 5 at stress levels of $348 \mathrm{MPa}$ resp. $463 \mathrm{MPa}$ show an increment in strain. Figure 6 shows the results of dilatometric compression tests where stress is plotted over strain. The shape of the MPEA5 stress-strain curve is similar to that from the in situ synchrotron compression test. The stress-strain curve of MPEA5 obtained in the dilatometer shows three positions where an increase in strain occurs. The increments in strain at the stress levels of $350 \mathrm{MPa}$ and $570 \mathrm{MPa}$ are nearly at the same stress levels as the strain-increments detected in the in situ compression test of 


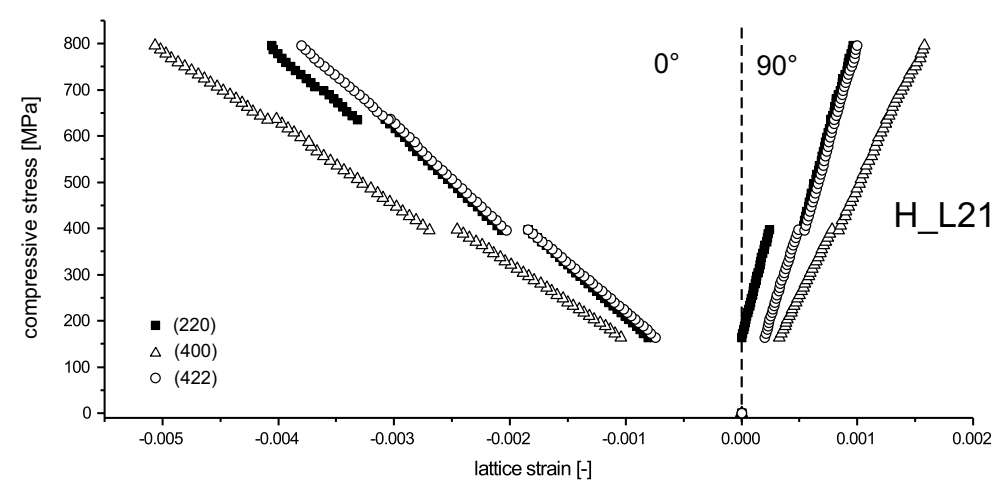

(a)

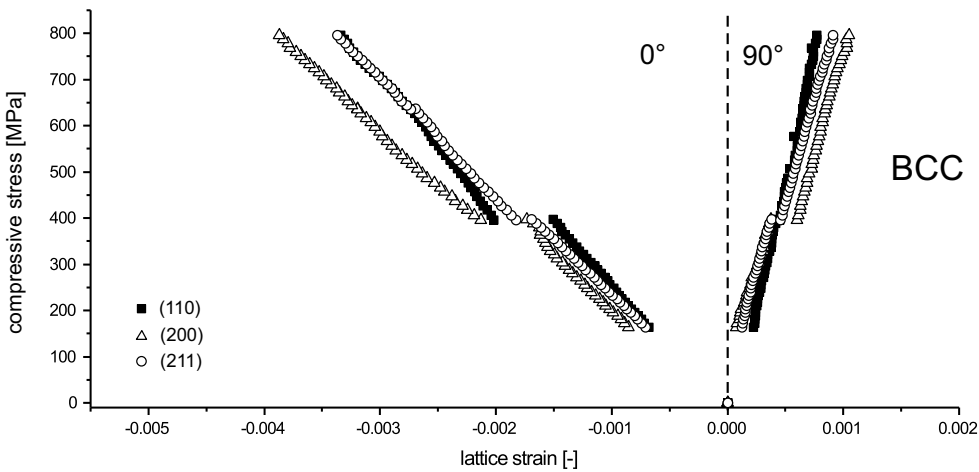

(b)

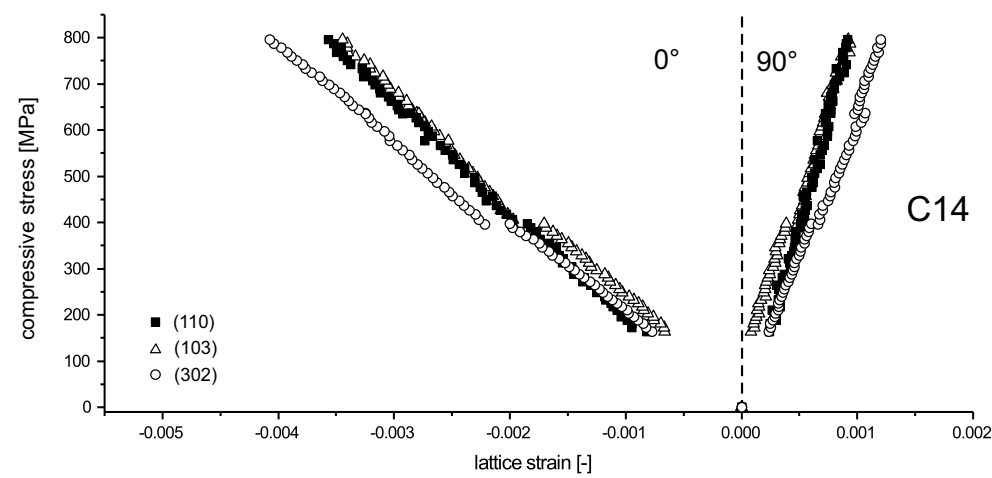

(c)

Fig. 4 Macroscopic stress plotted over lattice strain of composition MPEA_Ti10. Lattice strain is separated in parallel to loading direction $\left(0^{\circ}\right)$ and perpendicular to it $\left(90^{\circ}\right)$. The diagrams show results for H_L21-phase (a), BCC-phase (b) and C14_Laves-phase (c)

MPEA5. Due to the loading cell capability of the dilatometer application of higher compressive stresses than in the in situ synchrotron experiment (maximum applied load $\sim 635 \mathrm{MPa}$ ) was possible. At a stress level of $790 \mathrm{MPa}$, another increment in strain is detected in the dilatometer experiment.

The reason for the increase in macroscopic strain (in some cases associated with a drop in stress) and a decrease in the elastic modulus is a phenomenon which is most likely caused by the formation of long cracks [16-18]. This is supported by the observation that the MPEA5-samples used for the compression tests fracture during unloading after the maximum stress was applied. Since the macroscopic determination of the stressstrain curve is influenced by the entire isotropic material structure of the specimen, microstructural changes such as crack formation do not lead to any detectable change in the tangent modulus. The MPEA_Ti10 samples did not show any kinks in the stress-strain curves (Figs. 6 and 7) that would indicate the development of long 


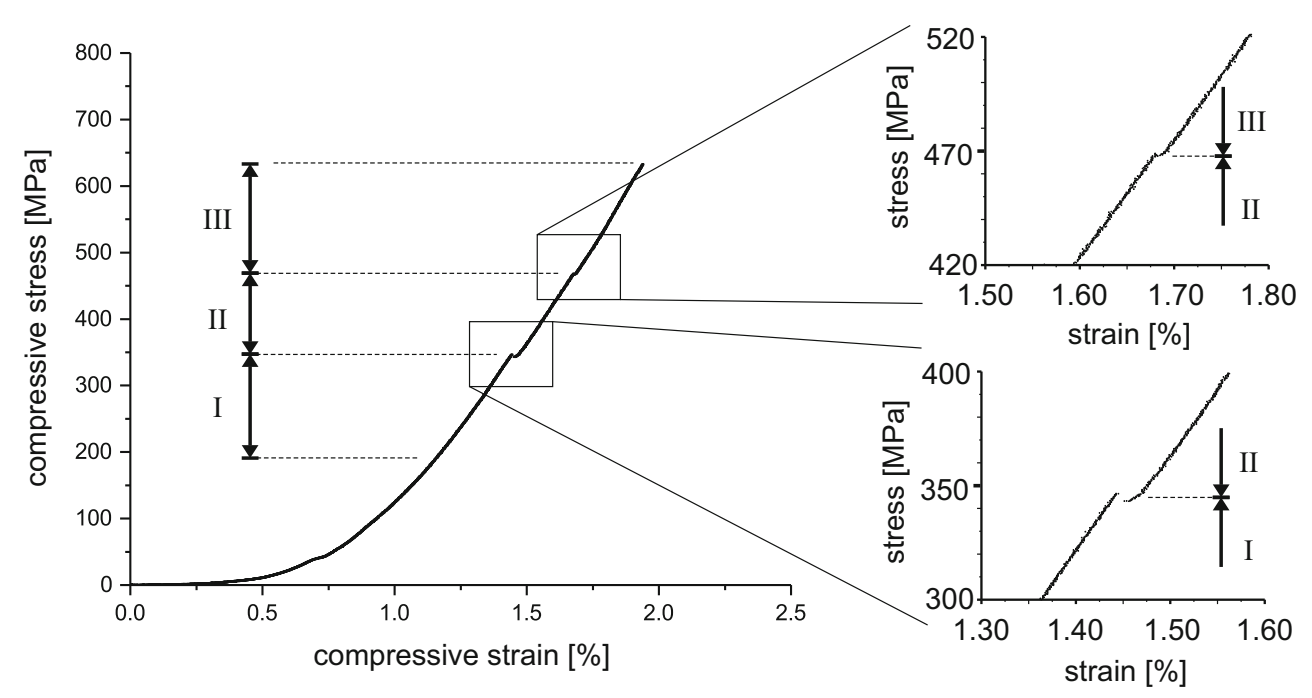

Fig. 5 Macroscopic stress plotted over strain of composition MPEA5 during the in situ synchrotron compression test. The portions of the curve, where discontinuities occur, are enlarged. The distinct parts of the curves are marked as I, II and III

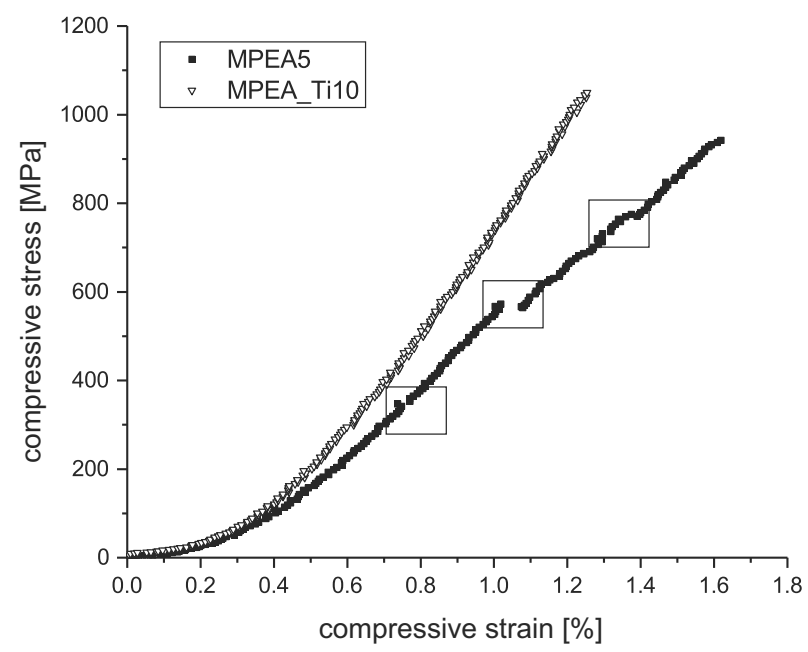

Fig. 6 Macroscopic stress plotted over macroscopic strain of compositions MPEA5 and MPEA_Ti10 determined from dilatometer tests at room temperature

cracks through the sample. Furthermore, the MPEA_Ti10 samples did not fracture into pieces during unloading at the end of the compression tests. Plastic deformation has not been observed in connection with the kinks.

A microscopic analysis of the samples after the compression tests showed cracks in both alloys (Fig. 8). While the cracks in MPEA_Ti10 developed with multiple branches (Fig. 8b), MPEA5 contains a continuous crack without branches (Fig. 8a). As branching is energetically inefficient for crack growth, crack propagation is hindered more than for continuous cracks [19-21]. This observation correlates to the stress-strain development of the alloys. Due to the interrupted growth of cracks forming branches in MPEA_Ti10, the stress-strain curve is only little affected.

\subsection{Detected phases in MPEA5 and MPEA_Ti10}

Figure 2 shows diffractograms of MPEA5 and MPEA_Ti10 obtained from samples in the absence of mechanical loads. The peaks which were used for analysis of the lattice strain during macroscopic compression of the samples are marked by the respective phase including the reflecting lattice plane. One of the detected phases is the Full Heusler-phase (H_L21-phase) whose diffraction peaks are labeled as (220), (400) and (422). Further 


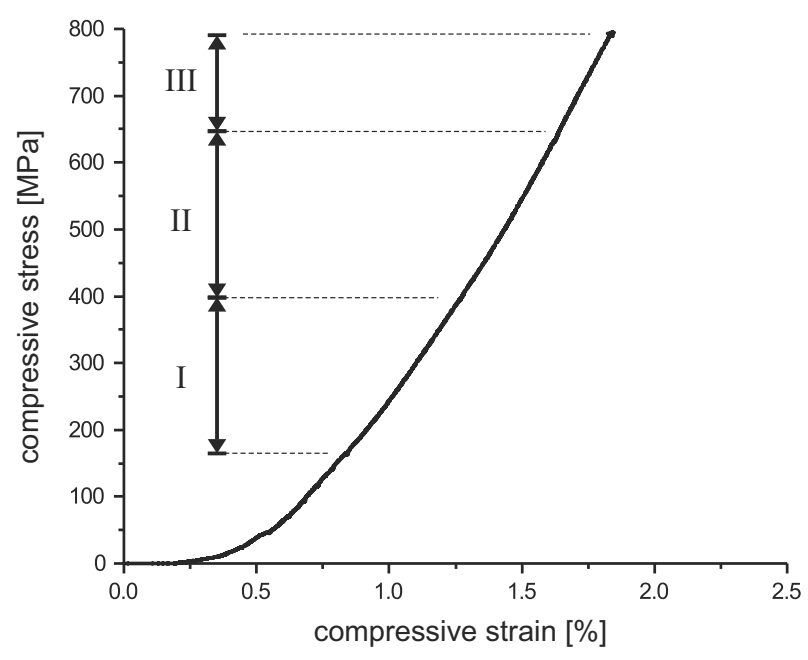

Fig. 7 Macroscopic stress plotted over macroscopic strain for composition MPEA_Ti10 during in situ synchrotron compression testing. The load stages of the experiment are marked by I, II and III
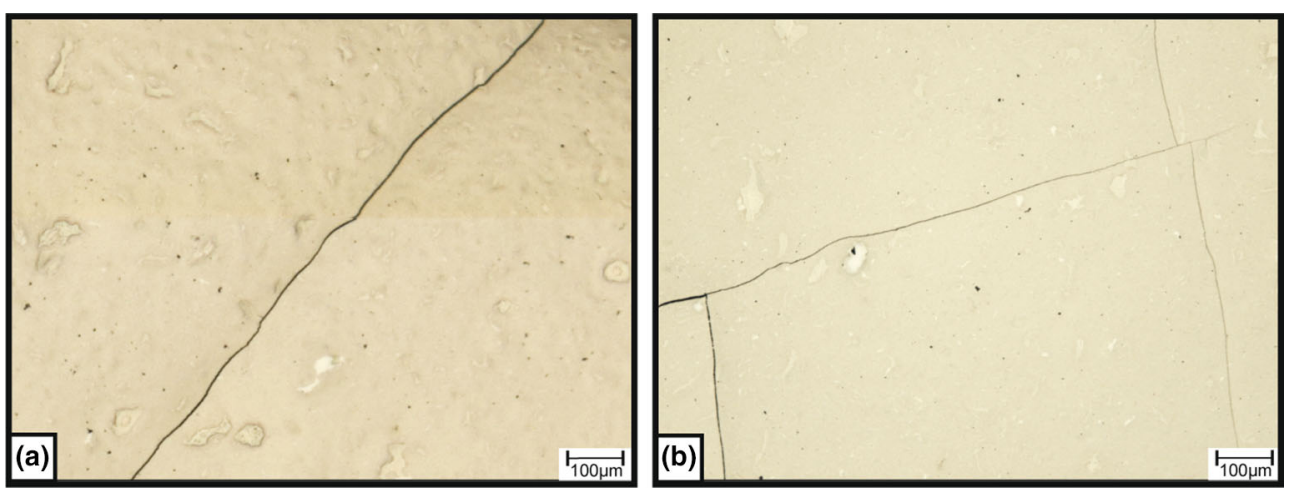

Fig. 8 Light microscopy images of metallography prepared cracked areas of MPEA5 and MPEA_Ti10. a Continuous crack in MPEA5. b Branching crack in MPEA_Ti10

peaks in the diffractograms indicate the presence of a body-centered cubic phase (BCC-phase). The reflecting lattice planes are (110), (200) and (211).H_L21-phase exhibits the peaks with the highest intensity, followed by peaks originating from the BCC-phase. As C14_Laves-phase is expected to be present only in small amounts, the according peaks in the diffractograms are small. The observed peaks for the C14_Laves-phase stem from the lattice planes (110), (103) and (302). The same phases observed in this work were also detected in X-ray diffraction experiments using an equimolar AlCrFeNiTi-alloy [10,12,22].

Thermocalc software predicts the presence of a H_L21-phase ( $\mathrm{Ni}_{2} \mathrm{AlTi}$ ) and several BCC-phases (Cr-rich, Fe-rich) in MPEA5 and MPEA_Ti10 (Fig.9). In MPEA5, C14_Laves-phase ( $\left.\mathrm{Fe}_{2} \mathrm{Ti}\right)$ is predicted to be not stable at room temperature but it should be present at temperatures above $130^{\circ} \mathrm{C}$. In MPEA_Ti10 a small amount of C14_Laves-phase is predicted to occur at room temperature. Probably due to a non-equilibrium state of MPEA5 and MPEA_Ti10, it is possible that a small amount of C14_Laves-phase is also detected at room temperature in MPEA5. Small peaks in the diffractograms indicating C14_Laves-phase support this assumption (Fig. 2).

Microstructure phase analysis via EBSD shows the presence of C14_Laves-phase ( $\left.\mathrm{Fe}_{2} \mathrm{Ti}\right)$ in both, MPEA5 and MPEA_Ti10 (Fig. 10c, d). Furthermore, BCC-phases (Fe-rich, Cr-rich) as well as H_L21-phase (Ni2AlTi) are detected. C14_Laves-phase agglomerates in areas with a maximum diameter of $1 \mu \mathrm{m}$. The distribution of BCC- and H_L21-phases is much finer. Figure 10c, d shows no correlation between crack propagation and microstructural phase distribution. Moreover, inverse pole figure maps (IPF-maps) of MPEA5 and MPEA_Ti10 (Fig. 10a, b) show, that the crack propagation in both alloys in general is intercrystalline. Only MPEA5 shows regions (about $2 \mu \mathrm{m}$ in size, circle in Fig. 10a) where crack propagation seems to be transcrystalline. 


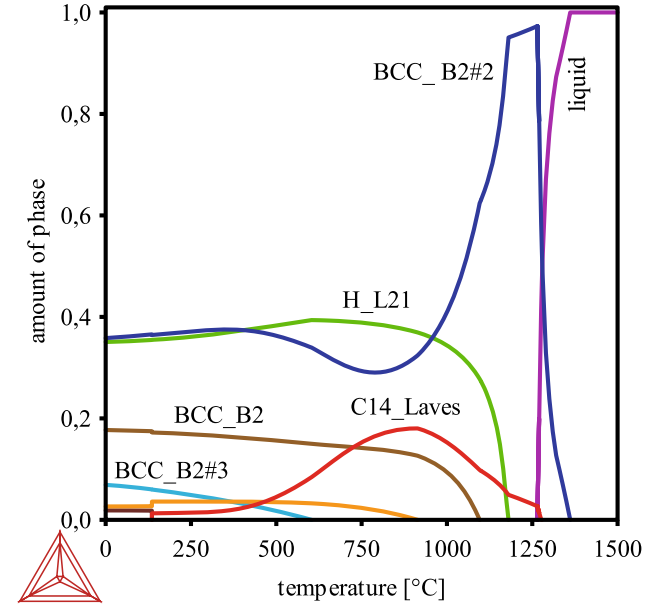

(a)

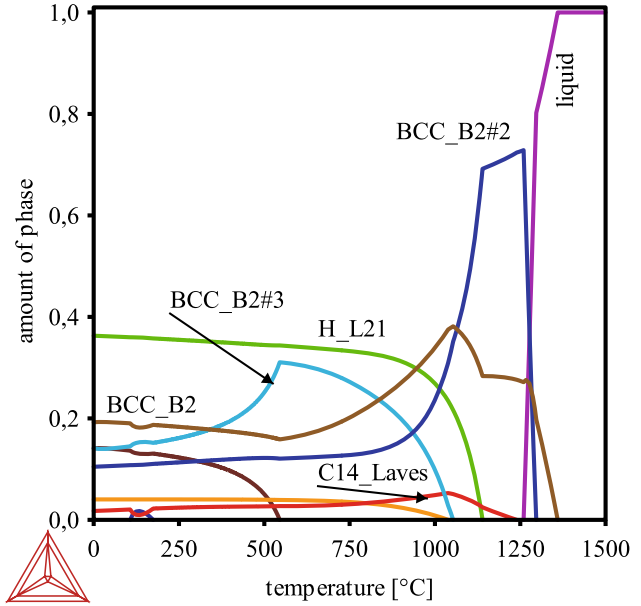

(b)

Fig. 9 Amount of stable phases in mole plotted over temperature according to calculation via Thermocalc for the compositions MPEA5 (a) and MPEA_Ti10 (b)
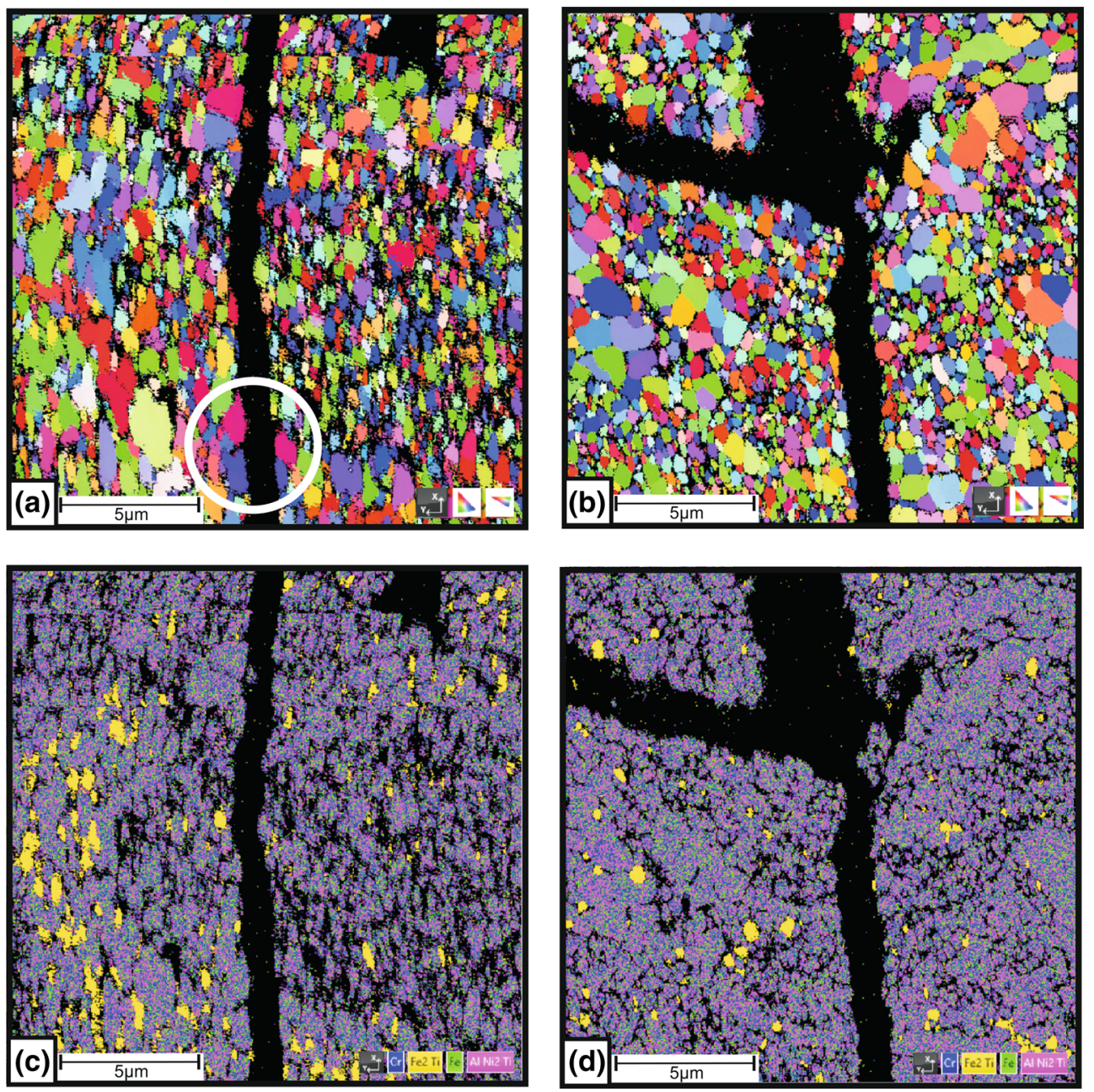

Fig. 10 EBSD images of cracked areas in MPEA5 and MPEA_Ti10. The upper images present IPF-maps of MPEA5 (a) and MPEA_Ti10 (b). The lower images show the phase distribution in MPEA5 (c) and MPEA_Ti10 (d). MPEA5 shows regions (circle in image a) where crack propagation seems to be transcrystalline 


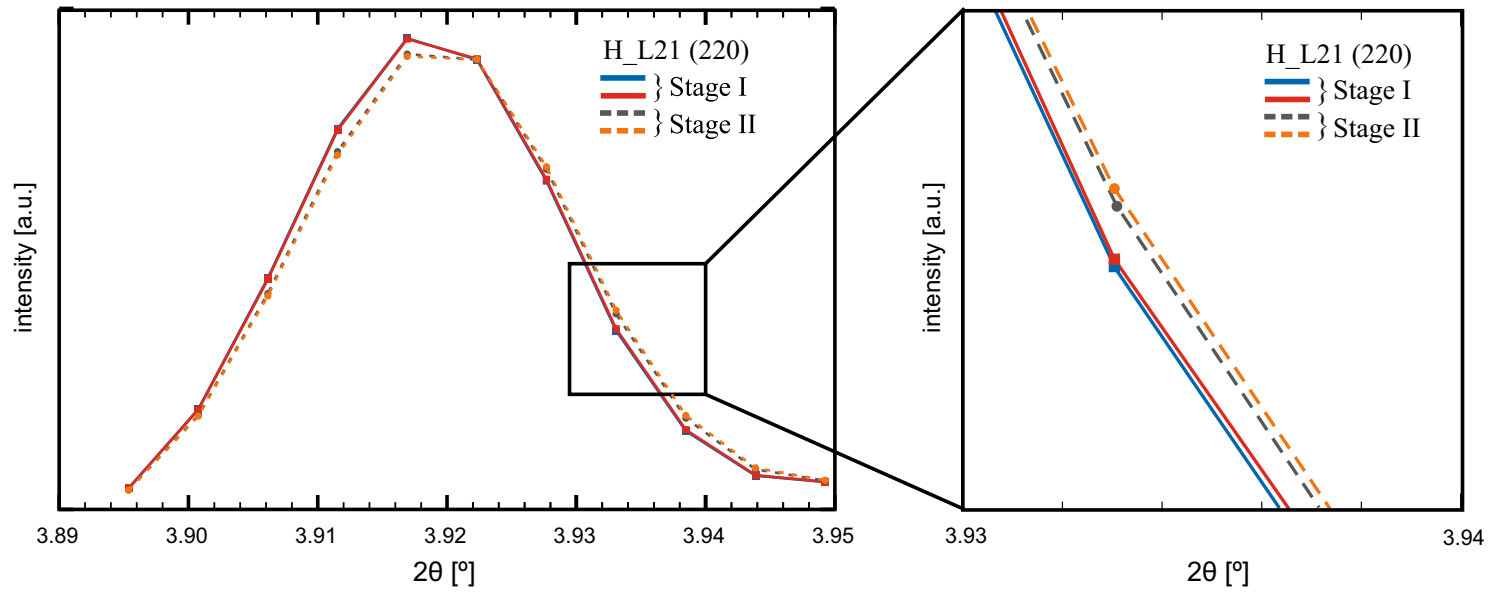

(a)

(b)

Fig. 11 Peaks evolution of H_L21-phase (220) in MPEA5. a The full line peaks represent the last two detections within stage I. The dotted line peaks represent the first two detections of stage II. b The enlarged area shows a gap between peaks of stage I and stage II

\subsection{Lattice-dependent elastic moduli as function of macro stress in MPEA5}

The analysis of lattice-dependent elastic moduli is separated into 3 regions depending on the applied macroscopic stress. As labeled in Fig. 5, stage I is defined up to the first major crack event (348 MPa), which can be detected from the stress-strain data. Stage II ranges between the first and second major crack events (348 MPa to $469 \mathrm{MPa}$ ). Stage III starts at a stress level of $469 \mathrm{MPa}$ and ranges up to the maximum applied stress of $636 \mathrm{MPa}$. The macroscopic stress is plotted over the calculated lattice strain (see Sect. 2.5) in Fig. 3. Thereby, different phases are presented separately $(a, b, c)$. The diagrams show results for distinct phase lattice planes and are presented for the loading direction $\left(0^{\circ}\right)$ as well as the direction perpendicular to it $\left(90^{\circ}\right)$.

Figure 11a shows diffraction peaks resulting from the lattice plane (220) of the H_L21-phase extracted from 4 diffractograms. The peaks represented by full lines stem from diffractograms which were taken just below the upper bound of stage I, whereas the dotted lines stem from the same peak slightly above the stress level at which stage II begins. The enlarged plot in Fig. 11b clearly shows that the gap between these two peaks just before the detected increment in strain is minimal. Also, the two peaks just after the detected increase in strain are nearly identical in position and shape. In contrast to this, the gap between the two pairs of peaks is clearly detectable. This results in distinctive increments in strain between stage I and stage II (Fig. 3). Having a look at the overall lattice-dependent elastic moduli, it is clearly visible that the value is reduced in later stages (Table 2). For example, the elastic modulus determined from lattice plane (220) of the H_L21-phase is $211.8 \pm 0.7 \mathrm{GPa}$ in stage I, and thereby reaches values that have been determined before in other studies (209.4 GPa [23], 193.0 GPa [24], 84.7-234.1 GPa [25,26]). In stage II, the lattice-dependent elastic modulus is about $28 \%$ lower and reaches $151.2 \pm 1.3 \mathrm{GPa}$. A further decrease in the lattice-dependent elastic modulus of over $6 \%$ is detected by reaching stage III $(141.8 \pm 1.1 \mathrm{GPa})$. This trend is shown by all lattice planes of all detected phases in the sample MPEA5. The BCC-phase shows lattice-dependent elastic moduli in stage I in the range of 232.3-301.5 GPa. These values have been confirmed before in other studies (Cr-rich BCC: 270-438 GPa [25,26], 289.6 GPa [27]; Fe-rich BCC: 233.3-287.6 GPa [25,26], 243.0 GPa [28]). The latticedependent elastic moduli of C14_Laves-phase are between 192.5 GPa and 207.2 GPa in stage I. Values close to this level have been determined in previous studies, as well (221-230 GPa [29], 179.0-265.0 GPa [25,26]). The decrease in lattice-dependent elastic moduli with increasing stage-number is supporting the assumption of the development of long, un-branching cracks between the single stages, as cracks decrease the materials' stiffness. Additionally, the increment in lattice strain between the stages supports the assumption of crack development, as crack development reduces the load-bearing cross section. This influences the lattice parameter. 


\subsection{Lattice-dependent elastic moduli as function of macro stress in MPEA_Ti10}

The diagrams in Fig. 4 present the macroscopic stress plotted over the lattice strain of composition MPEA_Ti10. The curves show increases in lattice strain at constant macroscopic stress levels of $398 \mathrm{MPa}$ and $636 \mathrm{MPa}$. In contrast to the increases of lattice strain in the composition MPEA5, here they do not correlate with crack development but with short stops at these loads during the test. The stops were used to perform texture measurements that revealed no noticeable change in texture in the sample. Creeping that occurs during the stops might be the reason for the increase in lattice strain. These positions were taken to divide the diagrams into 3 stages to compare the lattice-dependent elastic moduli at different stress levels. The stages are indicated in Fig. 7. Thereby, it has to be noted that the stress-strain curve progression in Fig. 7 does not give any indication of the stops in compression. Stage I is located between $167 \mathrm{MPa}$ and $398 \mathrm{MPa}$. Stage II is located between $398 \mathrm{MPa}$ and $636 \mathrm{MPa}$. Lastly, stage III is located between $636 \mathrm{MPa}$ and the maximum applied stress of $795 \mathrm{MPa}$.

In contrast to MPEA5, MPEA_Ti10 shows only little changes of the lattice-dependent elastic moduli in the different stages (Table 3). For example, the lattice-dependent elastic modulus determined from the reflection (220) of H_L21-phase in compression direction is $225.5 \pm 0.4 \mathrm{GPa}$. In stage II, the lattice-dependent elastic modulus is just $5 \%$ higher $(238.0 \pm 0.9 \mathrm{GPa})$. In stage III, the lattice-dependent elastic modulus is $206.7 \pm$ $3.0 \mathrm{GPa}(13 \%$ lower than in stage II). As the development of long, un-branching cracks is not the reason for the segmentation of the stress lattice strain curves of MPEA_Ti10, pronounced changes in lattice-dependent elastic moduli are not expected and hence are not detected.

\subsection{Lattice strain in dependence of phases in MPEA5 and MPEA_Ti10}

MPEA5 shows different lattice-dependent elastic moduli depending on the reflections investigated. For example, the lattice-dependent elastic moduli, determined from H_L21 reflections (220) and (422), are significantly higher than those determined from reflection (400). This implies that the lattice deformation during compression is larger in lattice direction [400] than in the lattice directions [220] and [422]. Comparing the reflections of the BCC-phase to each other, they result in nearly similar lattice-dependent elastic moduli. Still, there is a reflection identifiable in all stages that exhibits the minimal lattice-dependent elastic modulus. These are the (211) reflection in stage I, the (200) reflection in stage II and the (110) reflection in stage III even though the latticedependent elastic moduli determined from the two other reflections are just negligibly higher (+9\% for (200), $+11 \%$ for (211)). Determination of the lattice-dependent elastic moduli from reflections of the C14_Lavesphase is less accurate than for H_L21-phase and BCC-phase due to the small intensity and lower symmetry of the diffraction peaks originating from the C14_Laves-phase. The resulting lattice-dependent elastic moduli show no significant differences in dependence of the reflection. This indicates that the elastic deformation of the C14_Laves-phase is roughly the same in all observed lattice directions as determined from the reflections (110), (103) and (302). Comparing the lattice-dependent elastic moduli of H_L21- and BCC-phases shows that in general the elastic moduli of BCC-phase are significantly higher than those of the H_L21-phase. This means that the H_L21-phase takes a larger share of the total deformation during compression than the BCC-phase.

MPEA_Ti10 shows trends similar to MPEA5 in the development of phase-specific elastic moduli in dependence of the reflecting lattice plane.

The evolution of Poisson's ratio as a function of the stages varies depending on the phase considered. The deviation regarding Stage I for both compositions is between $-39.0 \%$ and $22.9 \%$ for BCC, between $-21.1 \%$ and $32.7 \%$ for C14_Laves and between $-1.5 \%$ and $10.2 \%$ for H_L21 (Tables 2,3). Therefore, H_L21 shows the smallest deviations of the Poisson's ratio. Further regularities or trends in the development of Poisson's ratio have not been detected.

\section{Conclusion}

MPEAs with equimolar concentration of the main elements $\mathrm{Al}, \mathrm{Cr}, \mathrm{Fe}, \mathrm{Ni}$ and $\mathrm{Ti}$ (MPEA5) as well as with a reduced amount of Ti (10 mol\%, MPEA_Ti10) were produced via mechanical alloying of powder then hot isostatic pressing. The dependence of macroscopic stress applied via compression tests on the evolution of lattice strain was detected via synchrotron X-ray diffraction at Beamline III of DESY [14]. MPEA5 shows the development of long, un-branching cracks with increasing compressive loading, which results in irregularities in the stress-strain behavior. 
Table 2 Lattice-dependent elastic moduli in loading direction $\left(0^{\circ}\right)$ and Poisson's ratio for MPEA5. Index $j$ identifies the stage (I, II, III)

\begin{tabular}{|c|c|c|c|c|c|}
\hline Phase & Plane & Stage & $E_{\mathrm{hkl}}(\mathrm{GPa})$ & $\left(E_{\mathrm{hkl}, j}-E_{\mathrm{hkl}, \mathrm{I}}\right) / E_{\mathrm{hkl}, \mathrm{I}}(\%)$ & $\nu_{\mathrm{hkl}}(-)$ \\
\hline \multirow[t]{9}{*}{ H_L21 } & \multirow[t]{3}{*}{220} & I & $211.8 \pm 0.7$ & 0.0 & $0.254 \pm 0.002$ \\
\hline & & II & $151.2 \pm 1.3$ & -28.6 & $0.265 \pm 0.012$ \\
\hline & & III & $141.8 \pm 1.1$ & -33.0 & $0.252 \pm 0.004$ \\
\hline & \multirow[t]{3}{*}{400} & I & $165.9 \pm 0.6$ & 0.0 & $0.305 \pm 0.002$ \\
\hline & & II & $128.1 \pm 1.1$ & -22.8 & $0.336 \pm 0.014$ \\
\hline & & III & $111.4 \pm 1.0$ & -32.8 & $0.317 \pm 0.006$ \\
\hline & \multirow[t]{3}{*}{422} & I & $206.1 \pm 0.7$ & 0.0 & $0.250 \pm 0.002$ \\
\hline & & II & $159.1 \pm 1.4$ & -22.8 & $0.274 \pm 0.013$ \\
\hline & & III & $143.6 \pm 1.2$ & -30.3 & $0.259 \pm 0.004$ \\
\hline \multirow{9}{*}{$\mathrm{BCC}$} & \multirow[t]{3}{*}{110} & I & $293.4 \pm 2.8$ & 0.0 & $0.372 \pm 0.008$ \\
\hline & & II & $202.7 \pm 2.1$ & -30.9 & $0.303 \pm 0.020$ \\
\hline & & III & $142.1 \pm 3.8$ & -51.6 & $0.237 \pm 0.011$ \\
\hline & \multirow[t]{3}{*}{200} & I & $301.5 \pm 4.0$ & 0.0 & $0.348 \pm 0.010$ \\
\hline & & II & $142.4 \pm 1.9$ & -52.8 & $0.212 \pm 0.019$ \\
\hline & & III & $156.0 \pm 2.0$ & -48.3 & $0.237 \pm 0.010$ \\
\hline & \multirow[t]{3}{*}{211} & I & $232.3 \pm 0.7$ & 0.0 & $0.285 \pm 0.003$ \\
\hline & & II & $182.0 \pm 1.3$ & -21.7 & $0.305 \pm 0.016$ \\
\hline & & III & $158.1 \pm 1.8$ & -32.0 & $0.260 \pm 0.008$ \\
\hline \multirow[t]{9}{*}{ C14 } & \multirow[t]{3}{*}{110} & I & $207.2 \pm 3.3$ & 0.0 & $0.314 \pm 0.029$ \\
\hline & & II & $148.9 \pm 9.8$ & -28.1 & $0.417 \pm 0.078$ \\
\hline & & III & $144.2 \pm 5.4$ & -30.4 & $0.375 \pm 0.089$ \\
\hline & \multirow[t]{3}{*}{103} & I & $195.0 \pm 1.8$ & 0.0 & $0.298 \pm 0.016$ \\
\hline & & II & $166.0 \pm 3.7$ & -14.9 & $0.338 \pm 0.039$ \\
\hline & & III & $137.0 \pm 2.6$ & -29.7 & $0.285 \pm 0.027$ \\
\hline & \multirow[t]{3}{*}{302} & I & $192.5 \pm 0.6$ & 0.0 & $0.283 \pm 0.003$ \\
\hline & & II & $143.4 \pm 1.6$ & -25.5 & $0.282 \pm 0.013$ \\
\hline & & III & $108.0 \pm 2.1$ & -43.9 & $0.223 \pm 0.009$ \\
\hline
\end{tabular}

Table 3 Lattice-dependent elastic moduli in loading direction $\left(0^{\circ}\right)$ and Poisson's ratio for MPEA_Ti10. Index $j$ identifies the stage (I, II, III)

\begin{tabular}{|c|c|c|c|c|c|}
\hline Phase & Plane & Stage & $E_{\mathrm{hkl}}(\mathrm{GPa})$ & $\left(E_{\mathrm{hkl}, j}-E_{\mathrm{hkl}, \mathrm{I}}\right) / E_{\mathrm{hkl}, \mathrm{I}}(\%)$ & $\nu_{\mathrm{hkl}}(-)$ \\
\hline \multirow[t]{9}{*}{ H_L21 } & \multirow[t]{3}{*}{220} & I & $225.5 \pm 0.4$ & 0.0 & $0.231 \pm 0.002$ \\
\hline & & II & $238.0 \pm 0.9$ & 5.6 & $0.255 \pm 0.002$ \\
\hline & & III & $206.7 \pm 3.0$ & -8.4 & $0.248 \pm 0.007$ \\
\hline & \multirow[t]{3}{*}{400} & I & $164.9 \pm 0.2$ & 0.0 & $0.321 \pm 0.002$ \\
\hline & & II & $180.7 \pm 1.0$ & 9.6 & $0.316 \pm 0.004$ \\
\hline & & III & $164.1 \pm 0.9$ & -0.5 & $0.344 \pm 0.006$ \\
\hline & \multirow[t]{3}{*}{422} & I & $211.6 \pm 0.4$ & 0.0 & $0.263 \pm 0.002$ \\
\hline & & II & $239.5 \pm 1.1$ & 13.2 & $0.262 \pm 0.003$ \\
\hline & & III & $211.5 \pm 1.3$ & -0.1 & $0.263 \pm 0.004$ \\
\hline \multirow[t]{9}{*}{$\mathrm{BCC}$} & \multirow[t]{3}{*}{110} & I & $283.5 \pm 1.5$ & 0.0 & $0.249 \pm 0.006$ \\
\hline & & II & $333.0 \pm 2.1$ & 17.5 & $0.306 \pm 0.015$ \\
\hline & & III & $257.5 \pm 3.4$ & -9.2 & $0.197 \pm 0.011$ \\
\hline & \multirow[t]{3}{*}{200} & I & $266.1 \pm 4.8$ & 0.0 & $0.352 \pm 0.012$ \\
\hline & & II & $224.9 \pm 3.1$ & -15.5 & $0.233 \pm 0.006$ \\
\hline & & III & $235.8 \pm 1.8$ & -11.4 & $0.308 \pm 0.009$ \\
\hline & \multirow[t]{3}{*}{211} & I & $238.2 \pm 0.4$ & 0.0 & $0.263 \pm 0.004$ \\
\hline & & II & $276.0 \pm 2.7$ & 15.9 & $0.304 \pm 0.005$ \\
\hline & & III & $250.8 \pm 1.8$ & 5.3 & $0.315 \pm 0.006$ \\
\hline \multirow[t]{9}{*}{ C14 } & \multirow[t]{3}{*}{110} & I & $235.3 \pm 3.7$ & 0.0 & $0.286 \pm 0.018$ \\
\hline & & II & $265.3 \pm 5.5$ & 12.7 & $0.308 \pm 0.024$ \\
\hline & & III & $242.7 \pm 6.7$ & 3.1 & $0.312 \pm 0.036$ \\
\hline & \multirow[t]{3}{*}{103} & I & $216.1 \pm 1.6$ & 0.0 & $0.276 \pm 0.008$ \\
\hline & & II & $283.4 \pm 3.7$ & 31.1 & $0.295 \pm 0.010$ \\
\hline & & III & $238.5 \pm 5.8$ & 10.4 & $0.336 \pm 0.031$ \\
\hline & \multirow{3}{*}{302} & I & $189.7 \pm 1.3$ & 0.0 & $0.303 \pm 0.005$ \\
\hline & & II & $229.1 \pm 1.6$ & 20.7 & $0.390 \pm 0.009$ \\
\hline & & III & $195.0 \pm 1.8$ & 2.8 & $0.282 \pm 0.011$ \\
\hline
\end{tabular}


Moreover, the lattice-dependent elastic moduli decrease after formation of cracks that are not perpendicular to the loading direction, as the load-bearing cross section becomes smaller. The lattice-dependent elastic moduli in loading direction $\left(0^{\circ}\right)$ are significantly lower than those in direction perpendicular to it $\left(90^{\circ}\right)$. MPEA_Ti10 shows the development of branching cracks during compression tests. As a result, only minor changes in latticedependent elastic moduli as a function of the applied compressive load are detectable. The reason for a higher resistance against propagation of long cracks in MPEA_Ti10 could be its lower amount of brittle C14_Lavesphase compared to that in composition MPEA5. In general, the BCC-phase shows higher lattice-dependent elastic moduli than the H_L21- and C14-phases. For this reason, the BCC-phase has a stronger resistance to deformation under compressive loading than the other phases in MPEA5 and MPEA_Ti10. Overall, elastic anisotropy was observed by analyzing the lattice-dependent elastic moduli of different reflections and phases. The detailed analysis of phase-specific mechanical properties in MPEAs enables their systematic optimization by reducing or increasing individual phase amounts. Further in situ synchrotron X-ray diffraction tests with adapted MPEA compositions are necessary to investigate in more detail the behavior of the phases present and their lattice strain under compressive loading.

Acknowledgements "We acknowledge DESY (Hamburg, Germany), a member of the Helmholtz Association HGF, for the provision of experimental facilities. Parts of this research were carried out at PETRA III."

Funding Open Access funding enabled and organized by Projekt DEAL.

Open Access This article is licensed under a Creative Commons Attribution 4.0 International License, which permits use, sharing, adaptation, distribution and reproduction in any medium or format, as long as you give appropriate credit to the original author(s) and the source, provide a link to the Creative Commons licence, and indicate if changes were made. The images or other third party material in this article are included in the article's Creative Commons licence, unless indicated otherwise in a credit line to the material. If material is not included in the article's Creative Commons licence and your intended use is not permitted by statutory regulation or exceeds the permitted use, you will need to obtain permission directly from the copyright holder. To view a copy of this licence, visit http://creativecommons.org/licenses/by/4.0/.

\section{References}

1. Miracle, D.B., Senkov, O.N.: A critical review of high entropy alloys and related concepts. Acta Materialia 122, 448-511 (2017)

2. Yusenko, K.V., Riva, S., Crichton, W.A., Spektor, K., Bykova, E., Pakhomova, A., Tudball, A., Kupenko, I., Rohrbach, A., Klemme, S., Mazzali, F., Margadonna, S., Lavery, N.P., Brown, S.G.: High-pressure high-temperature tailoring of high entropy alloys for extreme environments. J. Alloys Compd. 738, 491-500 (2018)

3. Murty, B.S., Yeh, J.-W., Ranganathan, S.: High-Entropy Alloys, vol. 1. Elsevier, Amsterdam (2014)

4. Special Materials Corporation.: Inconel alloy 718: datasheet SMC-045 (2007)

5. Reed, R.C.: The Superalloys: Fundamentals and Applications. Cambridge University Press, Cambridge (2006)

6. von Kobylinski, J., Lawitzki, R., Hofmann, M., Krempaszky, C., Werner, E.: Micromechanical behaviour of Ni-based superalloys close to the yield point: a comparative study between neutron diffraction on different polycrystalline microstructures and crystal plasticity finite element modelling. Continuum Mech. Thermodyn. 31(3), 691-702 (2019)

7. Mignanelli, P.M., Jones, N.G., Pickering, E.J., Messé, O., Rae, C., Hardy, M.C., Stone, H.J.: Gamma-gamma prime-gamma double prime dual-superlattice superalloys. Scripta Mater. 136, 136-140 (2017)

8. Böhler Edelstahl GMBH \& Co KG. Böhler W722 VMR: datasheet (2009)

9. Sedlak, J., Rican, D., Piska, M., Rozkosny, L.: Study of materials produced by powder metallurgy using classical and modern additive laser technology. Proc. Eng. 100, 1232-1241 (2015)

10. Reiberg, M., Kobylinski, J.V., Werner, E.: Characterization of powder metallurgically produced AlCrFeNiTi multi-principle element alloys. Continuum Mech. Thermodyn. 32(4), 1147-1158 (2020)

11. Nong, Z.-S., Lei, Y.-N., Zhu, J.-C.: Wear and oxidation resistances of AlCrFeNiTi-based high entropy alloys. Intermetallics 101, 144-151 (2018)

12. Chen, S.-K., Lee, P.-H., Lin, C.-H.: AlCoCrFeNiTi and its equal-molar five-component alloys in a metal mixological enthalpyentropy plane. Quart. Phys. Rev. 3(1), 1-28 (2017)

13. Liu, S., Gao, M.C., Liaw, P.K., Zhang, Y.: Microstructures and mechanical properties of $\mathrm{Al}_{\mathrm{x}} \mathrm{CrFeNiTi}_{0.25}$ alloys. J. Alloys Compd. 619, 610-615 (2015)

14. Schell, N., Martins, R.V., Beckmann, F., Ruhnau, H.U., Kiehn, R., Schreyer, A.: The high energy materials science beamline at PETRA III. Mater. Sci. Forum 571-572, 261-266 (2008)

15. Erdely, P., Staron, P., Maawad, E., Schell, N., Clemens, H., Mayer, S.: Lattice and phase strain evolution during tensile loading of an intermetallic, multi-phase $\gamma$-TiAl based alloy. Acta Mater. 158, 193-205 (2018)

16. Xue, L., Qi, M., Qin, S., Li, G., Li, P., Wang, M.: A potential strain indicator for brittle failure prediction of low-porosity rock: part II-theoretical studies based on renormalization group theory. Rock Mech. Rock Eng. 48(5), 1773-1785 (2015)

17. Xu, X., Wu, S., Jin, A., Gao, Y.: Review of the relationships between crack initiation stress, mode I fracture toughness and tensile strength of geo-materials. Int. J. Geomech. 18(10), 04018136 (2018)

18. Hoek, E., Martin, C.D.: Fracture initiation and propagation in intact rock-a review. J. Rock Mech. Geotech. Eng. 6(4), 287-300 (2014) 
19. Broek, D.: Elementary Engineering Fracture Mechanics. Springer, Amsterdam (1982)

20. Rösler, J., Harders, H., Bäker, M.: Mechanisches Verhalten der Werkstoffe. Springer, Fachmedien Wiesbaden (2012)

21. Cherepanov, G.P.: Methods of Fracture Mechanics: Solid Matter Physics. Solid Mechanics and Its Applications. Springer, Amsterdam (1997)

22. Wolff-Goodrich, S., Marshal, A., Pradeep, K.G., Dehm, G., Schneider, J.M., Liebscher, C.: Combinatorial exploration of B2/L2 1 precipitation strengthened AlCrFeNiTi compositionally complex alloys. SSRN Electron. J. (2019)

23. Wen, Z., Zhao, Y., Hou, H., Wang, B., Han, P.: The mechanical and thermodynamic properties of Heusler compounds $\mathrm{Ni}_{2} \mathrm{XAl}$ (X = Sc, Ti, V) under pressure and temperature: a first-principles study. Mater. Des. 114, 398-403 (2017)

24. Reddy, P.V., Sreenivasa, K.V.: Ab initio study of Fermi surface and dynamical properties of $\mathrm{Ni}_{2} \mathrm{XAl}(\mathrm{X}=\mathrm{Ti}, \mathrm{V}, \mathrm{Zr}, \mathrm{Nb}, \mathrm{Hf}$ and Ta). J. Alloys Compd. 616, 527-534 (2014)

25. de Jong, M., Chen, W., Angsten, T., Jain, A., Notestine, R., Gamst, A., Sluiter, M., Ande, C.K., van der Zwaag, S., Plata, J.J., Toher, C., Curtarolo, S., Ceder, G., Persson, K.A., Asta, M.: Charting the complete elastic properties of inorganic crystalline compounds. Sci. Data 2(1), 1-13 (2015)

26. Jain, A., Ong, S.P., Hautier, G., Chen, W., Richards, W.D., Dacek, S., Cholia, S., Gunter, D., Skinner, D., Ceder, G., Persson, K.A.: Commentary: The materials project: a materials genome approach to accelerating materials innovation. APL Mater. 1(1), 011002 (2013)

27. Edwards, A.R., Nish, J.I., Wain, H.L.: The preparation and properties of high-purity chromium. Metall. Rev. 4(1), $403-449$ (1959)

28. Zhang, H., Punkkinen, M.P.J., Johansson, B., Vitos, L.: Theoretical elastic moduli of ferromagnetic bcc Fe alloys. J. Phys. Condens. Matter 22(27), 275402 (2010)

29. Hoppe, V., Gibas, A., Grzegorczyk, W., Małecki, M., Hasiak, M.: Experimental study of Fe-Ni-Ti-Cr system. Interdiscip. J. Eng. Sci. 7(1), 1-9 (2019)

Publisher's Note Springer Nature remains neutral with regard to jurisdictional claims in published maps and institutional affiliations. 